\title{
INFLUENCIA DA MENSTRUAÇÃO SOBRE AS EPILEPSIAS
}

\author{
LUIS MARQUES-ASBIS *
}

É conhecido o fato do paciente epiléptico exibir certas características próprias no que se refere ao padrão das crises, à sua frequência e à localização no tempo. Classicamente, no que se refere aos fatores que influenciam a frequência dos ataques, à menstruação tem sido dado especial realce. No presente trabalho propomo-nos a estudar, do ponto de vista clínico, a influência da menstruação sobre as epilepsias.

\section{CASUISTICA E METODOS}

De um grupo de 3.457 pacientes epilépticas registrados no Ambulatório de Epilepsia da Divisáo de Neurologia do Hospital das Clinicas de São Paulo, foram separados 1.770 pacientes de sexo feminino. Destas, 1.574 tinham idade compreendida entre 12 e 50 anos, perído considerado como provável no que se refere à capacidade ovulatória da mulher (Tabela 1); 1.261 eram brancas, 110 pretas, 196 pardas e 7 amarelas. Na investigaçăo foram valorizadas a manifestaçoes clínicas, sendo dado espacial realce ds convulsóes. As pacientes foram estudadas do ponto de vista electrencefalográfico.

Procurou-se verificar a influencia que a menstruaçăo exercia sobre as crises convulsivas epilépticas, sendo destacado o aparecimento e/ou exacerbaçăo das crises nos perídos pré-menstrual, menstrual e pós-menstrual, sendo referidos também os casos que melhoraram das crises nesses períodos. As pacientes que sofreram influência da mens-

Idade (anos) Pacientes

$\begin{array}{rr}12-20 & 708 \\ 21-30 & 476 \\ 31-40 & 276 \\ 41-60 & 114 \\ \text { Total } & 1.574\end{array}$

Tabela 1 - Distribuicão etdria das pacientes com capacidade ovulatoria.

Trabalho da Clínica Neurológica do Departamento de Neuro-psiquiatria do Hospital das Clínicas da Universidade de Săo Paulo, apresenta:o no IX Congresso Brasileiro de Neurologia, realizado de 12 a 16 de outubro de 1980 em Curitiba: * Livre Docente da Clínica Neurológica. 
truaçăo foram comparadas com um grupo de epllépticos em geral, objeto de publicaçőes anteriores 11,12, no que se refere à idade de início da doença (Tabela 2) e ao padrăo electrencefalográfico (Tabela 4). No que se refere a frequência das convulsóes, as pacientes que sofreram influencia da menstruaçăo foram comparadas com as pacientes em idade ovulatória (Tabela 3). Finalmente foi registrada a frequência das convulsóes em 476 pacientes epilépticos de sexo masculino da mesma faixa etáría (12 aos 50 anos), sendo destaca jos os casos com incidencia mensal de convulsóes, ritmo que pode ser comparado ao periodo ovulatório da mulher.

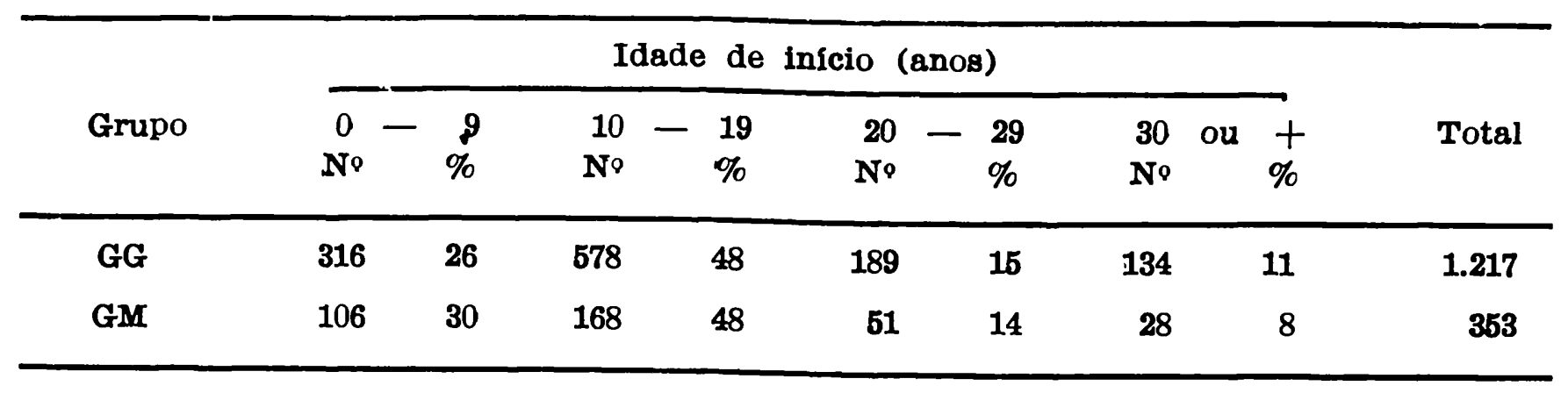

Tabela 2 - Idade de inicio da doenca no orupo que sofreu influencia da menstruagao (GM) comparado com um orupo de epilepsia em geral (GQ).

\begin{tabular}{|c|c|c|c|}
\hline Frequência (dias) & GO & GM & Total \\
\hline $\begin{array}{lll}1 / 1 & \text { a } & 7 / 7\end{array}$ & 138 & 62 & 200 \\
\hline $8 / \delta$ a $30 / 30$ & 262 (112) & 175 (74) & 437 (186) \\
\hline $31 / 31$ a $90 / 90$ & 110 & 29 & 139 \\
\hline$<90 / 90$ & 91 & 36 & 187 \\
\hline formas iniciais & 124 & 8 & 138 \\
\hline varí́vel & 220 & 43 & 863 \\
\hline Total & 945 & 353 & 1.298 \\
\hline
\end{tabular}

\footnotetext{
Tabela $s$ - Frequencia das convulsós nas pacientes que sofreram influencia da menstruagio (GM) relativamente ds que apresentavam idade ovulatónia (GO). Entre parentesis as que apresentaram frequencia mensal de crises.
}

\begin{tabular}{lrrrrr}
\hline & \multicolumn{2}{c}{ GG } & \multicolumn{3}{c}{ GM } \\
& No & $\%$ & No & $\%$ \\
\hline Normal & 367 & 30 & 107 & 30 \\
Difuso & 92 & 8 & 33 & 10 \\
Dpbs & 59 & 5 & 18 & 5 \\
Temporal & 487 & 40 & 103 & 29 \\
Focal e outros & 213 & 17 & 92 & 26 \\
\hline \multicolumn{2}{c}{ Total } & 1.217 & 100 & 353 & 100 \\
\hline
\end{tabular}

Tabela 4 - O EEG das pacientes que sofreram influencia da menstruacdo (GM) relativamente a um orupo de epilepsias em geral (GQ). 


\section{RESULTADOS}

Do grupo de 1.574 epilépticas em idade ovulatória, 121 apresentavam crises motoras năo convulsivas, 41 crises de automatismo, 158 crises sensitivo-sensoriais, 140 crises psiquicas e 143 crises autônomas; em 1.298 casos foram registradas convulsóes primárias ou secundárias. Fol observado que 353 pacientes com convulsão sofreram influencia da menstruaça (22,4\%). Fm 38 casos foram referidas melhoras no perfodo menstrual e, em 315, aparecimento e/ou exacerbaçăo das convulsóes. As pioras ocorreram imediatamente antes do periodo menstrual em 142 pacientes, durante em 44, imediatamente após em 36, antes e durante em 35, antes e ap 6 s em 33, durante e apos em 4 e antes, durante e após a menstruaçăo em 21 pacientes. A idade de inicio da doença das pacientes que sofreram influéncia da menstruaça relativamente ao grupo de epilepsia em geral pode ser observada na Tabela 2. No que se refere a trequencia das convulsóes (Tabela 3), 112 das pacientes em idade ovulatória apresentavam convulsర̃es mensais (12\%), enquanto tal frequência fol observada no grupo que sofreu influencia da menstruaça em 74 mulheres (22\%). O resultado do electrencefalograma das pacientes que sofreram influência da menstriagáo comparativamente com um grupo de epilepsia em geral pode ser observado na Tabela 4. Finalmente o levantamento feito em 476 pacientes epilépticos de sexo masculino da mesma falxa etária mostrou que 407 apresentavam convulsóes, dos quais em 66 a frequência das crises convulsivas era mensal (16\%).

\section{COMENTARIOS}

Em tratados clássicos de epilepsia tem sido destacada a sua forma menstrual ou catamenial. Lennox e Lennox ${ }^{6}$ encontraram alguma relação entre os dois eventos em 48,5\% de 656 pacientes de sexo feminino. Livingstone 8 também chamou a atenção para o problema em pacientes adolescentes. Pesquisa desenvolvida por Almquist 1 (1955) trouxe alguns abalos às teorias que correlacionavam epilepsia e menstruação; estudando 62 homens e 84 mulheres epilépticos no que se refere à periodicidade das crises, mostrou ritmo semelhante dos ataques em ambos os sexos, colocando em dúvida a idéia do ciclo menstrual como fator determinante de importância na ritmicidade das crises. Laidlaw 5 (1956) fez abordagem diferente do problema, estudando 50 pacientes epilépticas internadas, em intervalo de 25 anos, no que se refere à incidência de crises maiores relativamente à menstruação; o autor concluiu haver fortes ev́idências estatísticas de que há redução da freqüência das crises na fase luteínica, com aumento irregular imediatamente antes, durante e após a menstruação. Tenta explicar o fenômeno por provável ação anticonvulsiva da progesterona, com exacerbação das crises quando sua secreção cessa.

A partir daí vários trabalhos em animais 9 e na mulher 2,10 realçaram 0 papel que os hormônios desempenhariam sobre as epilepsias no período menstrual, sendo chamada a atenção para o efeito epileptogênico do estrogênio $e$ ação inibidora da progesterona. Assim sendo, teria importância na epilepsia catamenial a relação estrogênio/progesterona: quanto mais elevada, maior a suscetibilidade e vice-versa. O papel desempenhado pelos hormônios na epilepsia menstrual levou Wieczorek e col. ${ }^{13}$ a testar a ação de anticoncepcionais na evolução das epilepsias menstruais, não tendo sido observados efeitos benéficos; pelo contrário, houve pioras em alguns casos. 
Das 1.574 pacientes epilépticas estudadas no presente trabalho, em 22,4\% foi observada influência da menstruação ou, pelo menos, alteração do ritmo das crises nesse período. As pioras observadas predominaram nos períodos pré-menstrual e menstrual. Por outro lado, relativamente à idade de início da epilepsia (Tabela 2), quanto mais avançada a idade, menor a chance de haver influência da menstruação. O estudo da frequência das convulsões (Tabela 3) mostrou que a incidência mensal nas pacientes que sofreram influência da menstruação (22\%) foi quase o dobro da observada nas pacientes em idade ovulatória (12\%). Tal achado é compreensível, pois corresponde às epilépticas que apresentaram convulsões apenas no período menstrual; daí se infere que as pacientes em idade ovulatória que apresentaram convulsões mensais e que não foram incluídas nas que sofreram influência da menstruação, apresentaram ritmicidade semelhante, porém com incidência mensal de crises fora do periodo menstrual. Cumpre notar que o estudo do grupo de pacientes em idade ovulatória, independentemente de terem sido ou não influenciadas pcla menstruação, mostrou incidência mensal de crises em $14 \%$ dos casos. Por outro lado, levantamento feito em 476 de sexo masculino da mesma faixa etária, dos quais 407 apresentaram convulsões, evidenciou que em 66 casos a frequência das crises era mensal (16\%). Evidentemente no homem não há $c$ ciclo biológico que caracteriza a mulher em idade ovulatória. Portanto a comparação do ritmo mensal de crises no sexo masculino (16\%) com o mesmo ritmo nas mulheres (14\%), considerados ambos na mesma faixa etária, não mostra diferenças significativas. Tal fato depõe a favor dos achados de Almquist ${ }^{1}$ sendo lícito, a partir dos dados fornecidos pela presente investigação, colocar em dúvida a correlação entre dois eventos cíclicos, quais sejam, as crises epilépticas e os períodos menstruais.

As pesquisas electrencefalográficas referidas na literatura $3,4,7$ referem-se à atividade bioelétrica cerebral durante o período menstrual. $O$ estudo do padrão electrencefalográfico de nossas pacientes relativamente a um grupo de epilépticos em geral (Tabela 4) mostrou menor predominância das disritmias temporais nas pacientes cujas crises se exacerbaram no periodo menstrual e maior incidência de outras anormalidades focais.

A propósito dos fatores que podem influenciar a exacerbação das crises epilépticas no período menstrual, além do papel desempenhado pelos estrógenos e pela progesterona, como foi referido acima, não se pode desprezar a possibilidade dos hormônios interferirem no metabolismo das drogas antiepilépticas explicando, pelo menos em parte, esses fenômenos.

\section{RESUMO}

De um total de 3.457 pacientes epilépticos foram estudados clinicamente 1.574 mulheres em idade ovulatória. Os resultados obtidos permitiram ao autor chegar às seguintes conclusões: 1) Em 353 pacientes (22,4\%) as epilepsias sofreram influência da menstruação. 2) A exacerbação das crises predominou nos períodos pré-menstrual e menstrual. 3) $O$ índice percentual das pacientes em idade ovulatória e com frequência mensal de crises comparado com os 
mesmos indices de pacientes de sexo masculino da mesma faixa etária, não mostrou diferenças significativas; tal fato coloca em dúvida a correlação entre crises epilépticas, de um lado, e ciclo menstrual, de outro. 4) Nas pacientes que sofreram influência da menstruação houve predomínio das disritimias focais não temporais.

\section{SUMMARY}

\section{The influence of menstruation on the epilepsies.}

From 3.457 epileptic patients, 1.574 females from 12 to 50 years old, considered probably in ovulatory age, were studied regarding the influence of menstruation upon epileptic fits. The females were compared with a group of epilepsies in general and with a group of male epileptic patients (476 cases) of same age exhibiting monthly seizures, a rhythm similar to the menstrual cycle in woman. The results led the author to the following conclusions: 1) In 353 epileptic females $(22,4 \%)$ the epilepsy was influenced by the menstruation. 2) The exacerbation of fits predominate in the premenstrual and menstrual periods. 3) No significant differences was observed when compared females in ovulatory age exhibiting monthly seizures (14\%) with male of similar age and similar frequency of seizures $(16 \%)$; this fact is against the correlation between the cyclical events represented by epileptic fits and menstruation. 4) Focal non-temporal abnormalities predominate in the females that was influenced by menstruation.

\section{REFERENCIAS}

1. ALMQVIST, R. - The rhythm of epileptic attacks and its relationship to menstrual cycle. Acta psychiat. neurol. scand., 1955 (supl. 105).

2. BACKSTRON, T. - Eplleptic seizures in woman related to plasma estrogen and progesterone during menstrual cycle. Acta. neurol. scand. 54:321, 1976.

3. CRHUTZFELDT, O. D.; WILHELM, H. \& WUTTKE, W. - EEG changes during spontaneous and controled menstrual cycles and their correlation with psychological performance. Electroencephal. Clin. Neurophysiol. 40:113, 1976.

4. GAUTRAY, J. P.; GARREL, S. \& FAU, R. - Electroencephalographic correlates of the human menstrual cycle. II - Results and discussion. Acta Furop. Fert. 2:15, 1970. Resumo em Epilepsy Abstracts 4:146, 1971, ref. 826.

5. LAIDLAW, J. - Catamenial epilepsy. Lancet 271:1235, 1956.

6. LENNOX, W. G. \& LENNOX, M. A. - Epilepsy and Related Disorders. LittleBrown Co., Boston, 1860.

7. LIN, T. Y.; GREENBLATT, M. \& SALOMON, H. C. - A poligraphic study of one case of petit mal epilepsy: effects of medication and menstruation. Electroencephal. Clin. Neurophysiol. 4:351, 1952. Resumo em Epilepsy Abstracts 1:417, 1947/1967, ref. 2935.

8. LIVINGSTONE, S. - Comprehensive Management of Epilepsy in Infancy, Childhood and Adolescence. Charles C. Thomas, Springfield (Illinois), 1972. 
9. LOGOTHETIS, J.; HARNER, R.; MORREL, F. \& TORRES, F. - The role of strogens in catamenial exacerbations of epilepsy. Neurology (Minneapolis) 9:352, 1959.

10. LONGO, L. P. \& SALDANA, L. E. G. - Hormones and their influence in epilepsy. Acta Neurol. Latinoemer. 12:29, 1966.

11. MARQUES-ASSIS, L. - Consideracões a propósito do tratamento medicamentoso de 1217 pacientes epilépticos. I - Estudo em relaçăo ao típo de epilepsia e ao electrencefalograma. Arq. Neuro-Psiquiat. (Såo Paulo) 27:312, 1969.

12. MARQUES-ASSIS, L. - Tratamento medicamentoso de 1217 pacientes epllépticos. II - Estudo em relaçăo à idađe de início, ao tempo de doença e a frequencia das crises. Arq. Neuro-Psiquiat. (São Paulo) 28:44, 1970.

13. WIECZOREK, V.; BQCK, R. \& KLUGE, H. - The use of ovulation inhibitors in female epileptics and psychiatric patients. Munch. Med. Wschr. 111:254, 1969. Resumo em Epilepsy Abstracts 2:97, 1969, ref. 597.

Clinica Nemrolbgica - Fraculdade de Medicina da Universidade de sdo Paulo Caisa Postal 3461 - 01000 8øo Paulo, 8P - Brasil. 